\title{
Integrated IT Platform for Coordination of Diagnosis, Treatment, and Aftercare of Prosthetic Joint Infections
}

\author{
CHRISTIAN SUREN $^{1}$, SUSANNE FEIHL ${ }^{2}$, CHRISTIANE QUERBACH ${ }^{3}$, PATRICK RÄMER ${ }^{2}$, \\ FLORIAN POHLIG ${ }^{1}$, JAKOB THURNER $^{1}$, RUDOLF BERNARD ${ }^{3}$, DIRK BUSCH ${ }^{2}$, \\ RÜDIGER VON EISENHART-ROTHE ${ }^{1 *}$ and HEINRICH MARIA LAURENTIUS MÜHLHOFER ${ }^{1 *}$ \\ ${ }^{1}$ Department for Orthopedics and Sports Orthopedics, ${ }^{3}$ Apotheke, Klinikum rechts der Isar, \\ Technical University Munich, Munich, Germany; \\ ${ }^{2}$ Institute for Medical Microbiology, Immunology and Hygiene, Technical University Munich, Munich, Germany
}

\begin{abstract}
Background/Aim: Prosthetic joint infections (PJI) are difficult to diagnose and treat. For a correct diagnosis, an array of information has to be processed and weighted. Successful treatment depends on the diagnosis, timing, and surgical strategy paired with treatment of the infectious agent. The complexity and interdisciplinarity needed cause difficulties concerning decision-making, the communication between disciplines, and the execution of a treatment strategy. The aim of this study was to develop a software platform to enhance the collection of information for the diagnosis of PJI, the interdisciplinary decision-making process, the communication between team members, and continuous evaluation of treatment. Patients and Methods: In regular planning sessions with an information technology (IT) specialist, a concept for an IT solution was chosen and the tool was designed in an interdisciplinary approach. Results: The tool has been used as a trial version since June 2017. It consists of 14 user interfaces with 431 items. A total of 117 patients with 118 infections have been entered and the strategy decided upon and communicated using 298 infection board documents outlining the treatment. The tool is now being used to organize the infections board agenda, schedule patient case discussions, document the relevant data and treatment plan, as well as communicate with the other teams
\end{abstract}

This article is freely accessible online.

*These Authors contributed equally to this study.

Correspondence to: Christian Suren, Klinik für Orthopädie und Sportorthopädie, Klinikum rechts der Isar, Technische Universität München, Ismaninger Str. 22, 81675 Munich, Germany. Tel: +49 8941402271, Fax: +498941404849, e-mail: christian.suren@ mri.tum.de

Key Words: Infection board, interdisciplinary, antibiotic stewardship, prosthetic joint infections, PJI. involved in the treatment. Conclusion: Using the developed tool enables the infections team to work collaboratively and under division of labor on each case, rendering the work flow more efficient for each team member.

Prosthetic joint infection (PJI) remains one of the most devastating complications after joint replacement. With the expected increase of the number of arthroplasties performed in an aging population $(1,2)$, PJI will remain a challenging complication for the treating physician on several levels:

First, as there is no single parameter proving or refuting the presence of PJI, the diagnosis depends on the correct interpretation and weighting of a variety of patient history data, clinical symptoms, laboratory findings, imaging, and pathological and microbiological results. Despite efforts by professional associations including the American Academy of Orthopaedic Surgeons (AAOS), the European Bone and Joint Infection Society (EBJIS), and the Musculoskeletal Infection Society (MSIS), as well as by organizations such as the Centers for Disease Control and Prevention (CDC), there is no uniform diagnostic approach (3-8). Therefore, the diagnosis of PJI, especially in centers treating highly complex cases, depends on the ability of an interdisciplinary team of experts to take into account a wide array of information and weight the findings according to the available evidence and personal experience.

Second, even though surgery remains paramount to the treatment of PJI, it is likely to fail unless it is part of a multifaceted strategy. Again, a team of experts from different fields should be involved in the development and execution of such a strategy. Moreover, the evidence available is scarce, as there are few level-one studies available (9-13). Finally, the increasing complexity of the diagnostic and therapeutic approaches at hand and the interdisciplinary effort itself pose challenges. The information that clinical decisions are based on is dispersed across a variety of sources both digital and analog. Electronic health records 
(EHR) often do not facilitate the extraction and summarization of particular findings for an interdisciplinary approach as is needed in the case of PJI. The gathering of all the necessary findings from paper records, electronic sources, or imaging and laboratory software for a joint decision is tedious and prone to mistakes and omissions. Another weak link in the process is the way that diagnosis and treatment decisions are communicated, whether that be among the team of experts or between them and other individuals or teams involved in the day-to-day treatment.

The said complexity of the diagnosis and treatment of PJI also impedes the follow-up of patients after treatment and the collection and stratification of the data needed to evaluate, compare, and improve treatment strategies.

To tackle the challenges arising on the fields of data gathering and stratification, communication among the decision makers and between them and other medical personnel, and the continuous collection and evaluation of relevant information during and after treatment, was the aim of our efforts described in the following.

\section{Patients and Methods}

Preliminary evaluation. At our Institution, which is a tertiary referral center for revision arthroplasty, there is a weekly conference of the Department of Orthopedics, the Institute of Microbiology, and the hospital pharmacy, the latter two forming the antibiotic stewardship (ABS) liaison for the entire hospital, on cases of PJI. For each case, the available diagnostic findings and previous as well as current therapies are discussed and evaluated by the members of this musculoskeletal infections team, and the therapeutic strategy as well as the kind and duration of anti-infective treatment are determined. Up to the beginning of this project, the results of such conferences were summarized in a text document distributed on the wards for the treating physicians and nurses to take note of. Upon discharge or transfer of a patient, the treatment plan and duration of treatment were transferred from this document into the discharge note, by hand, by the discharging physician. On follow-up visits, the discharge note in the hospital's EHR was used to assess the adherence to the planned treatment, and another electronic note in the EHR was made to document the current findings on each visit. At the start of the project, the participants of the conference each identified parts of the process that they considered to be unnecessarily complicated, to cause loss of information, and/or to enable mistakes and omissions. In an additional biweekly meeting after the conference, their opinions were gathered, discussed and summarized as follows.

Patient history and external diagnostic findings. Especially in cases with previous revisions, the relevant aspects of the patient history are dispersed among paper records, surgical reports, and discharge or short notes from other hospitals, electronic records from outpatient visits to our institution, and implant and allergy passes. External laboratory and arthrocentesis findings, microbiological culture and pathology results, and previous radiological studies are often gathered sequentially at outpatient contacts in our institution's clinic. The quality and completeness of the information transferred onto the digital EHR are variable. Paper copies are added to the paper file and are not accessible digitally within the timeframe of treatment, as the digitalization of paper records is regularly performed after discharge.

Internal diagnostic findings. While all diagnostic results at our institution are documented digitally, they are formatted as texts in the instances of pathology and microbiology results, surgical reports, discharge and short notes, and imaging reports. The texts cannot be searched systematically (for the number of granulocytes per high power field in pathology reports, for example). Furthermore, there is no option to visualize some selective pieces of information from different documents simultaneously.

Treatment strategy and follow-up. The surgical strategy (implant retention, single -, two -, or multi-stage implant exchange, implant removal) is determined by the type and duration of symptoms, and an array of objective findings. The type and duration of antiinfective medication complements the respective strategy. After consideration in the weekly conference, the strategy was communicated by means of a text document on the hospital server, as there was no suitable format within the EHR software. While sufficient in most standard cases, this procedure has the potential for mistakes and omissions when some objective results that required a change in strategy became available only after the conference, or when a change of strategy, or a deviation from the standard strategy in selected cases, needed to be communicated to other parties involved in the treatment.

The means for documentation of follow-up visits are short notes in the EHR. Comments on the adherence to the planned treatment, or on changes in antibiotic treatment after discharge, are stated in the text. As the notes are not searchable, bringing this information up again for later consideration is tedious. For statistical analysis, the data has to be transcribed manually into another format.

Visualization. Having the possibility to visualize a case and its pertinent information and findings, preferably over time, was expected to be advantageous both during and after discussion of the case in the conference.

Collaboration. As different findings become available over a period of time, the team members should be able to collaborate on the same case separately and preferably online.

Platform development and design. After the evaluation phase, an experienced clinical IT specialist was assigned to the musculoskeletal infections team to assess the team's suggestions for feasibility and start off with the project's design phase. The options considered were:

1. A solution within the existing EHR software consisting of the design of customized short notes and other documents for better synopsis of the relevant data. This approach would have entailed a dependence on the currently used EHR software, and the risk of having to start the process over with another software in case of a change of contract.

2. Search for and acquisition of existing software solutions for this purpose. As of the beginning of the development phase, there was, to our knowledge, no preexisting software with the required features. 3. Design and development of a customized platform as a standalone software solution. This approach was considered to be too time consuming and too demanding on personnel and resources. 


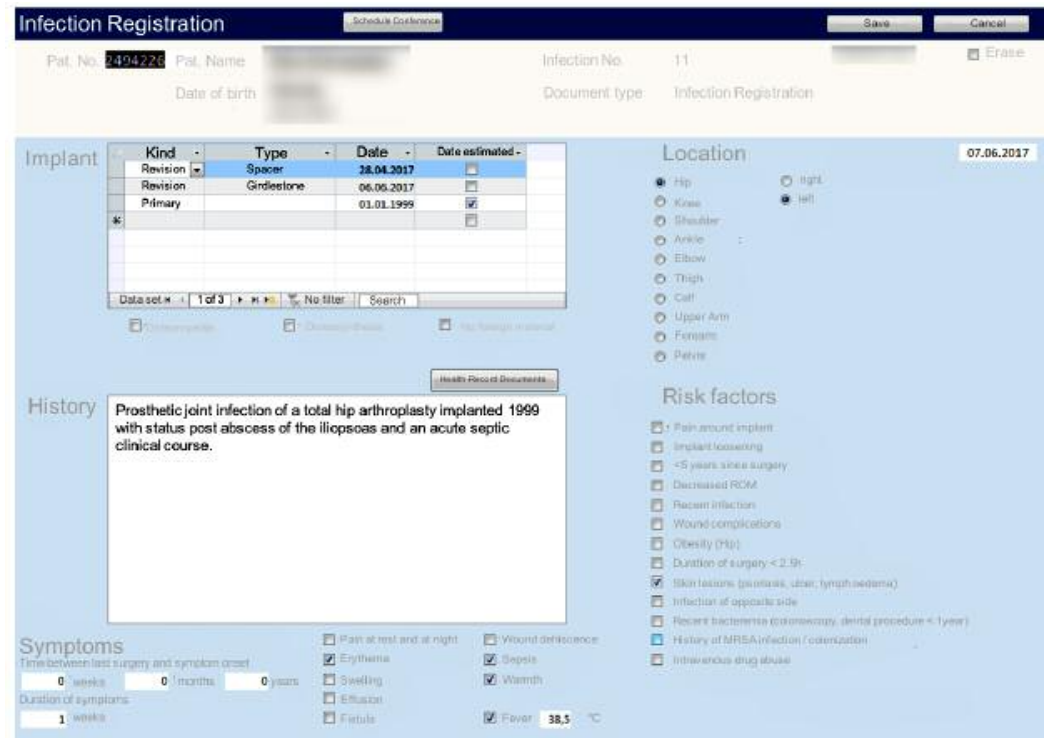

Figure 1. Infection registration document. Top row (dark blue bar): Document name, button to create an infection board document, "save" button, "cancel" button. Below (bright grey): patient number, patient name, patient date of birth, infection number, document identification. Below (light blue) from top left clockwise: Implant history table with implant type (primary, revision, megaprosthesis), date of surgery and whether the date is exact or by month/year. Below the table optional information: osteomyelitis, osteosynthesis in situ, and no implant in situ. Localization of implant (by joints and bones), date of the document's creation. Risk factors such as previous infection, status post bacteremia, loose implant. Patient history with button to access the EHR directly from the interface. Bottom: Symptoms such as swelling, redness, tenderness, duration of symptoms and time since last surgery.

4. Design and development of a customized platform within an existing software solution.

After separate meetings of each of the members of the musculoskeletal infections team with the IT specialist to discuss specific demands on the software solution, it was decided to create a customized platform within Microsoft Access (Microsoft Corporation, Redmont, WA, USA). Each member of the team was required to provide specific demands on the platform's content and user interface for their respective fields of expertise. These drafts were presented during the biweekly planning sessions and submitted to feedback by the other team members. The revised drafts were then assessed and incorporated into the platform's conceptual design by the IT specialist. His draft was yet again presented on a regular basis to the whole team and amended according to the feedback given.

Based on these drafts, several documents within Microsoft Access were designed to serve multiple functions: as data entry masks, as a pathway through our diagnostic and therapeutic algorithm, and as tools to assess the "filtered-down" relevant data. The data structure is centered around the infection itself, and patient data and documents are attributed to that infection. This enables the platform to separately characterize more than one infection in the same patient.

Infection registration document. Thus, the core element of the data document hierarchy in the Microsoft Access platform is the document for infection registration. The user allocates a certain patient via name and date of birth to the existence of an infection. The patient's personal data is pulled from the EHR automatically. Then, the following details characterizing the infection are entered (Figure 1):
Implant history: The date of implantation, the kind of implant (primary/revision/tumor prosthesis) and presence of an osteosynthesis or other foreign material are chosen by a drop-down menu.

Patient history regarding the infection and risk factors: This is followed by a free text section to enter the key points of the patient's reported history and complaints. The onset and duration of clinical symptoms as well as objective findings such as the temperature on admission or the presence of a fistula are documented as well as several risk factors for PJI (Figures 2 and 3).

In this document, the patient can be scheduled for evaluation in the weekly infection board meeting via a button that triggers the creation of an infection board document and a dialog with several other documents that can be checked or unchecked by the user. These documents include a strategy, surgery, arthrocentesis/biopsy, pathology, radiology, microbiology, anti-infective treatment, and follow-up document. Based on the user's choice, these are created within Access and are subordinate hierarchy elements of the infection board document.

In the strategy document, the planned course of treatment is outlined by a combination of menu items, including implant retention, single, two-stage, or multiple stage implant exchange, or implant removal. This document can also be used to describe a change of strategy, i.e. from implant retention to two-stage exchange.

In the surgery document, the date and kind of surgical procedure performed can be chosen in a similar fashion from a menu, including irrigation and debridement, prosthesis removal and implantation of a spacer, and resection arthroplasty.

The arthrocentesis/biopsy document allows users to enter the kind of diagnostic procedure performed including details such as the amount of synovial fluid drawn, the cell count within the fluid, and the amount and location of biopsies taken. 


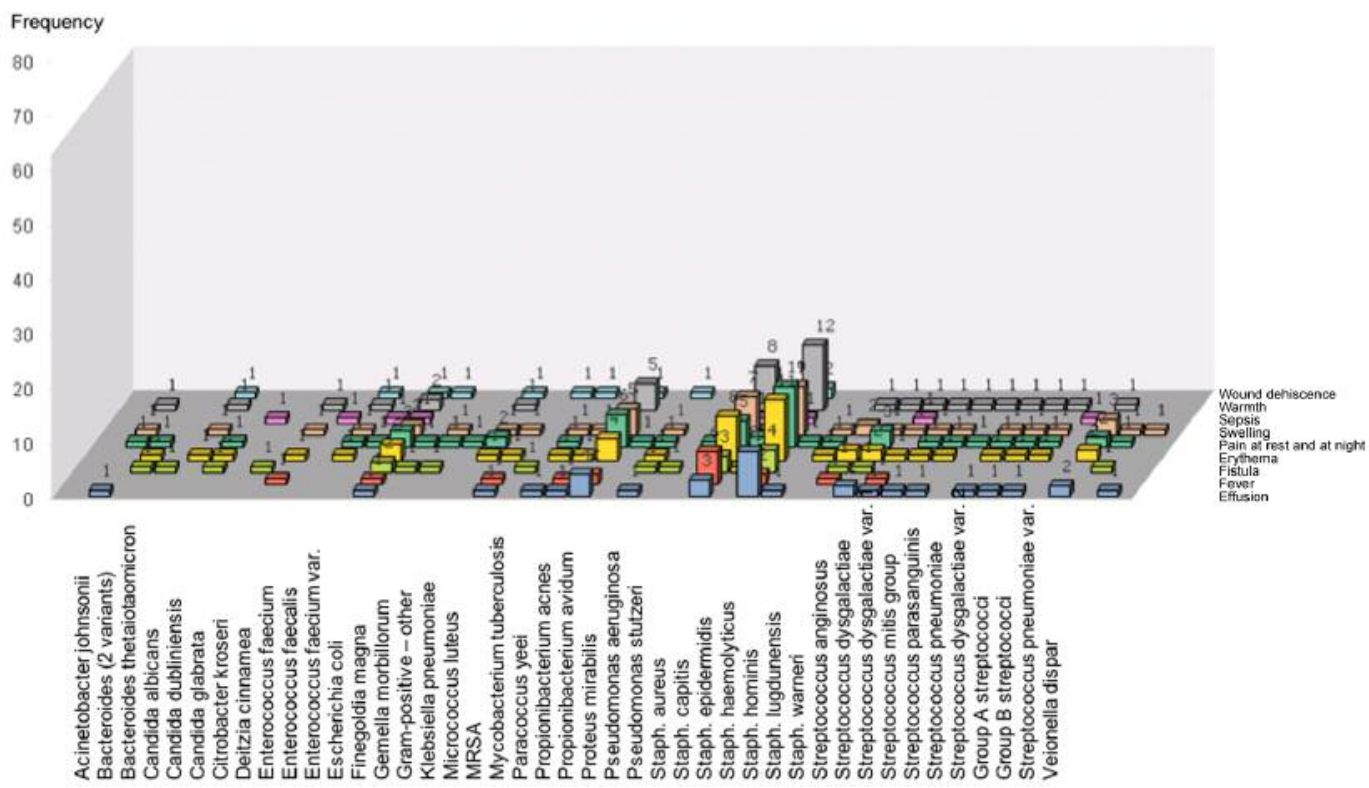

Figure 2. Example of real-time data analysis using Qlikview: $X$ axis: Type of bacterium, y axis: Number of observations, $z$ axis: Clinical symptoms. Clicking on a bar will show the respective cases in which the symptom was observed.

Similar to the above, the pathology document characterizes the pathological findings for the biopsies taken. Regarding the microbiology document, the presence and resistogram of infectious agents are entered, allowing a simultaneous visualization of every agent found for said infection or said patient.

The anti-infective treatment document holds the current treatment, possible interactions with the patient's regular medication, and ABS interventions. The treatment decided upon by the infection board is entered including its planned duration in days.

Upon follow-up, the patient's adherence to or a deviation from the planned treatment can be noted in the follow-up document. From this document, the user can access the anti-infective treatment document to make adjustments and can create a new strategy document to note a resulting change of strategy. Also, the patient can be scheduled for an infection board meeting by a button.

Infection board document. This document is designed as a control, documentation and communication tool for infection board meetings. It consists of an active and a passive section. The passive section includes the contents of the subordinate documents described above. On the other hand, the active section contains the current reason for presentation, the board's assessment on whether an infection is present, suggested further diagnostics, and a course of action. In the passive section, findings such as the arthrocentesis result can be checked to be entered into the active section.

To facilitate the simultaneous assessment of several laboratory results over time, a button in the passive document section triggers access to the QlikView server (QlikTech International, Radnor, PA, USA) showing an overview of selected findings (Figure 2). These are sorted in profiles. For example, one profile shows only inflammation markers, while another one relevant for the ABS team shows kidney and liver function parameters. These profiles can be combined, and the profiles' filters can be changed in real time (Figure 4).
Upon completion, the team can finalize the document, triggering the creation of a PDF file (Portable Document Format, Adobe Systems Incorporated, San José, CA, USA) containing a summary of the active document section. This document is saved to the patient's EHR, where it is instantly accessible by other individuals involved in the treatment, and it is thus the means of real time communication between the infection board and other medical personnel.

Infection board documents pending decision are shown in and can be accessed from a list which is now being used as the infection board meeting agenda. When findings such as the definitive culture results are not yet available, the patient's presentation is rescheduled to a future meeting by creating another infection board document for that date via a button.

Timeline. The individual sequence of events for each infection can be visualized on a Gantt chart (Varchart XGantt, Netronic, Aachen, Germany), where symbols mark laboratory results, clinical findings, surgeries, infection board decisions, and antibiotic treatment (Figure 5).

\section{Results}

From September 2016 until June 2017, the design phase was completed. 14 user interfaces were created in Access, containing a total of 431 items. An XGantt diagram was customized to visualize each infection over time, with symbols representing each document and a symbol for each laboratory investigation undertaken. Users can click on each symbol to pull up the details of the respective document.

Since June 2017, the platform has been in use for weekly infection board meetings. On a monthly basis, the developing IT specialist has been attending meetings for technical 
assistance and troubleshooting and to assess possible design amendments suggested by team members. The following workflow has been established to achieve adequate usage of the platform with optimal efficiency: Upon admission, patients with PJI are reported to a study nurse attached to the musculoskeletal infections team. The study nurse extracts relevant data from paper files and the EHR to complete the infection registration, and creates an infection board document scheduled for the upcoming meeting; then, the study nurse prepares the surgery, arthrocentesis/biopsy, radiology, and pathology documents. An orthopedic infection team member checks the prepared documents and signs them off electronically. The microbiologist of the team creates a microbiology document and updates culture results over time, the pharmacist documents the current medication and possible interactions, as well as resulting ABS interventions in an anti-infective treatment document.

During the weekly meeting, the patients pending for infection board assessment are chosen from the list of infection board documents to be completed. The findings entered by the team members are pulled up and discussed, with relevant findings selected for transfer into the active document section. Further instructions are entered as a free text if needed. If relevant findings are not yet completed, a preliminary course of treatment is set, signed off to create the PDF document for the EHR. The patient's presentation at a future meeting is scheduled by creating a new infection board document dated in the future. On the wards and in clinic, physicians involved in the patient treatment can access the PDF file via the EHR instantaneously.

Since implementation of the platform, 117 patients with 118 infections have been entered. A total of 298 infection board documents were filled out in 45 infection board meetings. There were 65 low grade infections, 35 early onset, and 23 acute hematogenic PJIs. The difference between the absolute number of infections and the sum of the infection types results from the fact that early onset or acute hematogenic infections can persist and be classified differently over the course of time or be acute on chronic manifestations of a low-grade infection undiagnosed at the first presentation of the patient (Figure 6).

\section{Discussion}

This is, to our knowledge, the first project aiming to integrate the relevant findings for the diagnosis and treatment of PJI and to enable a more efficient and complete communication among specialist team members and between them and other treating physicians.

Since most medical personnel are neither trained in the use of advanced IT applications nor in their development, we acquired professional assistance by an IT specialist and aimed for a structured and cyclical development process

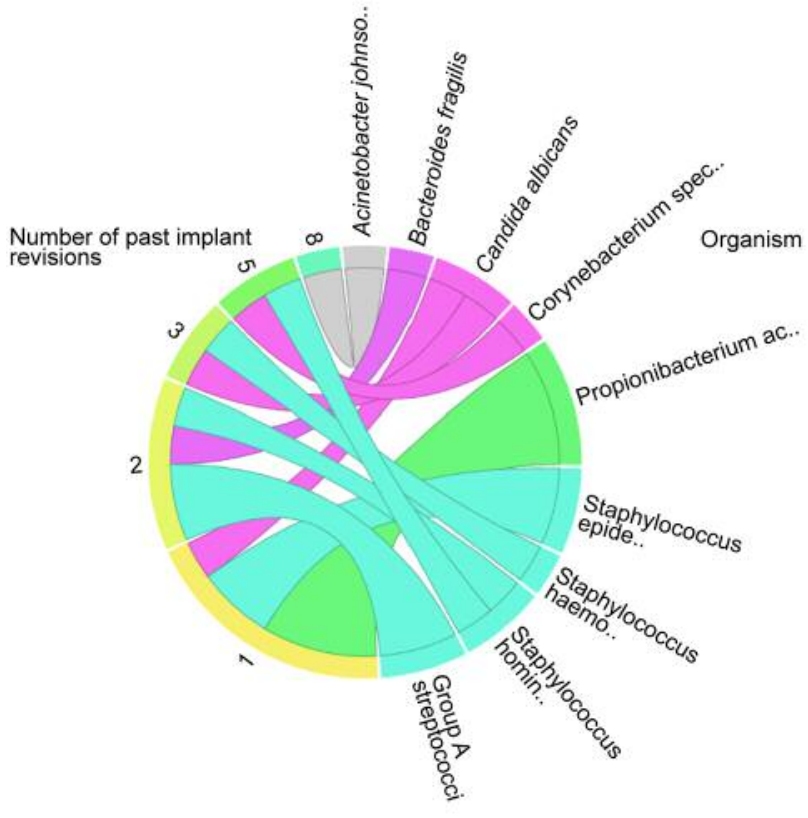

Figure 3. Example of real-time data analysis using Qlikview: Chord diagram. On the left: Number of previous surgeries. On the right: microorganism found. Width of the chord connecting the number of surgeries and the organism indicates the number of cases. Clicking on any element of the diagram shows all the cases pertaining to it. Additional filters (i.e., time of the index or any other surgery, the location of the prosthesis, or a restriction to certain organisms) can be set in real time.

involving recurrent checks and feedback. As this process was developed "on-the-go" by our team, one might speculate that the incorporation of an established, predefined workflow, as suggested by Collins et al. for collaborative creation of content, might have been less time consuming (14).

Also, our decision to use an established yet dated application such as Access should be critically discussed. More advanced software solutions might have offered more possibilities in terms of interface design, visualization, and automatic extraction and algorithmic processing of the raw data. Such amenities remain our mid-term goal. However, our strategy was to develop the created tool as a beta version and transfer it to a more modern software platform once it has been tried and tested. This way, we were able to focus our resources on the creation of content and a design suitable for the clinical and scientific purpose we pursued. The Access platform provided the necessary flexibility for quick amendments when changes of content or the user interface were deemed necessary by team members.

The value of an interdisciplinary approach in the diagnosis and treatment of infections is known $(15,16)$. The observation of organizational and communication problems in our practice prompted us create a customized solution 


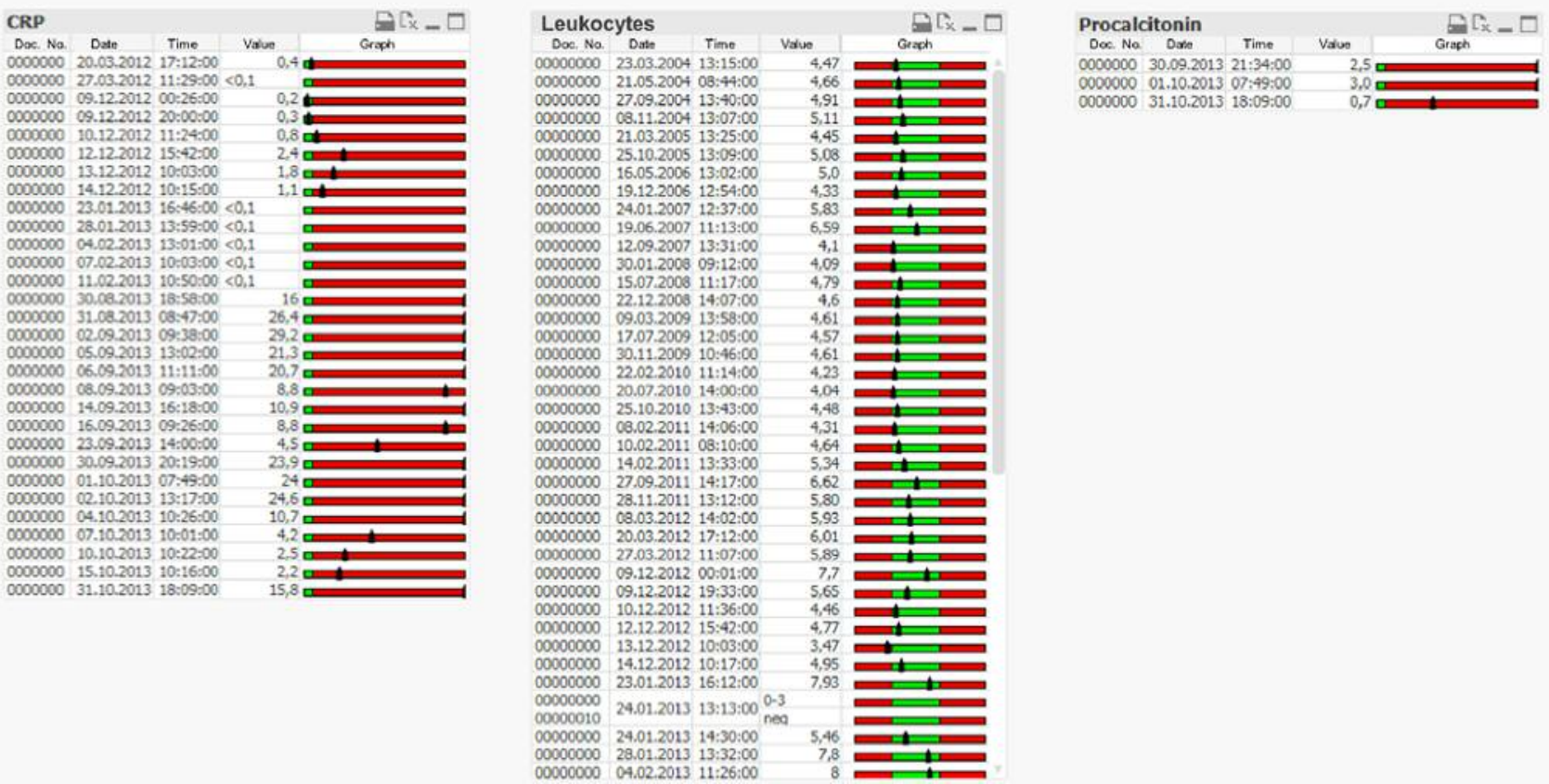

Figure 4. Laboratory results grouped using Qlikview: Parameters and time frame can be added in real time and saved as a preset. Shown here is part of the "infection" preset showing C-reactive protein (CRP), white blood cell count, and procalcitonin levels and ranges.

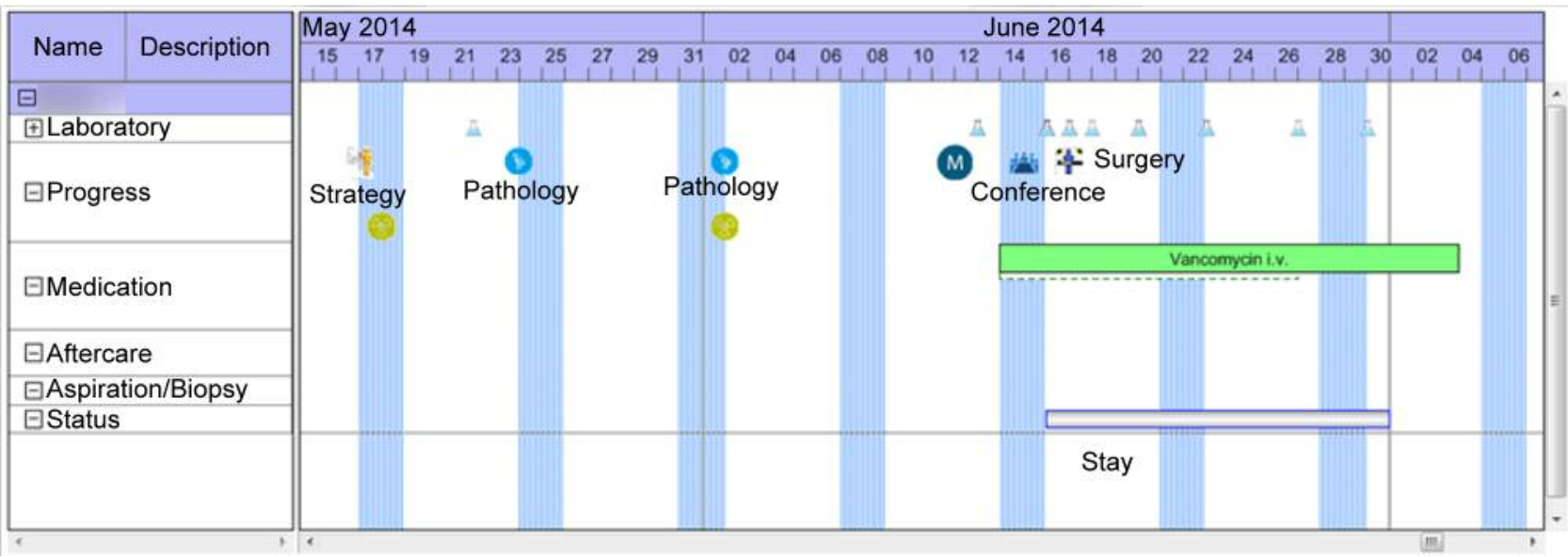

Figure 5. Gantt diagram showing the course of events over time. Symbols from left to right: Strategy document created. Yellow circle: Biopsy document. Light blue cone: Laboratory result. Blue circle: Pathology result. Dark blue circle "M": Microbiology result. Bar "Vancomycin i.v.": Duration and type of anti-infective treatment. Grey bar below: Duration of inpatient treatment at our institution. Each document or laboratory result can be accessed by clicking on the respective symbol.

with the added benefit of fully embracing information technology, since the dispersal of relevant information among various analog and digital sources has been identified as a hindrance to treatment quality and cost effectiveness $(17$, 18). Evans et al. have shown elegantly the improvements in antibiotic drug choice and usage as well as in cost effectiveness when the potential of information technology is put to use (17).

However, data quality has to be ensured by those collecting the data. Relevant professional terms have to be defined, data entry has to be consistent to enable later access and searchability, and entries have to be checked before they are 


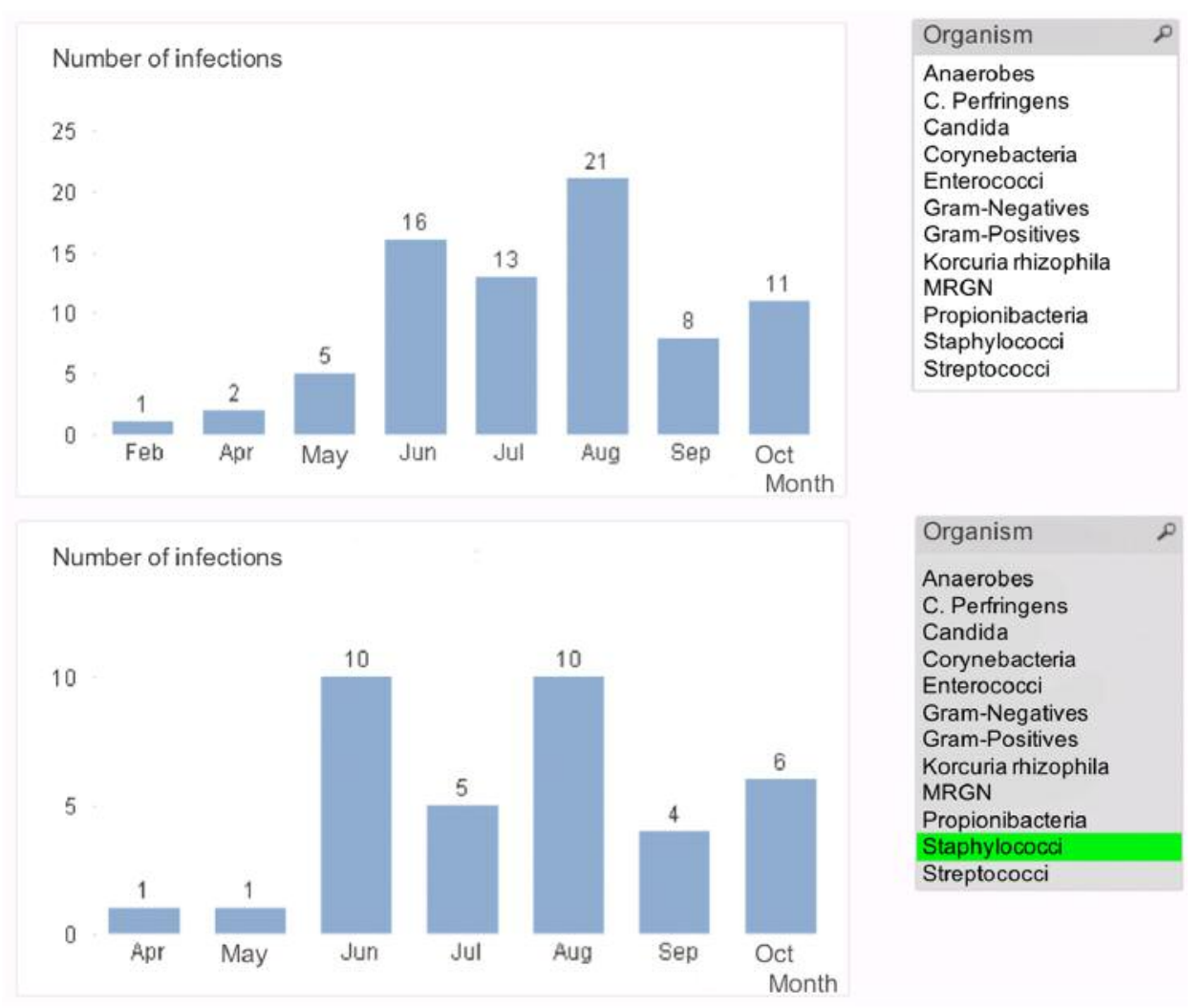

Figure 6. Example of real-time data analysis using Qlikview: Bar chart. X-axis: Months. Y-axis: Number of infections. Box on the right: Infectious agents detected. The upper chart shows an overview of all infections over a selected time span. Upon selection of one or more infectious agents (in this case, staphylococci), the chart changes to show cases of infections caused by staphylococci. Clicking on a bar will list all respective cases.

saved or transferred to other platforms (19). With our choice of customized user interfaces in Microsoft Access, we tried to put the selection and normalization of the relevant data, the most time-consuming part of the process in our experience, at the beginning of the workflow. Upon completion of the acquisition of relevant data after a patient is admitted, subsequent evaluation of the information is very straightforward. Therefore, the bulk of the time taken up for the preparation of the information for infection board meetings takes place outside of the team meeting. The meetings themselves have become more efficient in the process. Also, since the implementation of the platform, a substantially improved organization of infection board meetings and of the communication between the team members has been observed by all. A similar effect was described by Marks et al. after introduction of a database for infectious diseases (15).

Another potential benefit of the platform described is the possibility for remote access and data entry. Thus, the platform can be used to organize clusters of primary and secondary health care facilities organized around a center specialized in PJI diagnosis and treatment. Remote diagnosis and treatment assistance is necessary in a field with a high degree of specialization paired with an increasing financial burden caused by an increasing amount of PJI cases. A similar network approach has proven efficient, cost-effective and successful in the treatment of severe injuries in Germany, and studies on telemedicine reach similar conclusions (20-23). In the case of PJI, there is the additional potential benefit of providing remote care for outpatients, as the duration of antibiotic treatment often exceeds 2 months, and the tailored choice of anti-infective agents and management of side effects are challenging for general practitioners. Consistent reports on side effects and interactions observed in outpatient treatment could, in turn, provide antibiotic stewardship teams with valuable information and data, again improving antibiotic prescription and usage.

With the ongoing use of the platform, the quantity of data entered will likely enable a solid scientific evaluation of current diagnostic and treatment concepts. Due to the heterogeneous approaches to the definition, diagnosis, and treatment of PJI, and the relatively small numbers of patients treated in single institutions, there is a lack of adequately powered, well-executed trials on the subject. A positive effect of large amounts of data recorded through a network 
approach on the quality of scientific assertions on rare medical conditions has been shown in trauma research (24).

Inconsistencies and deviations from standardized diagnostic and clinical pathways for PJIs even in specialized high-volume centers have been described $(25,26)$. While there is no evidence for worse treatment outcomes associated with such deviations, an effect on overall treatment success is very likely. Apart from that, there is a moral obligation to try to reduce or better, eliminate mistakes during treatment of each patient in our care. The platform we developed now allows us to constantly evaluate the diagnostic and therapeutic processes, identifying systematic errors and improving our implemented pathways.

\section{Conclusion}

For diseases that are difficult to diagnose or treat, a collaborative approach involving a team of specialists in various fields is often needed. This is the case for prosthetic joint infections, were an expert team of orthopedic surgeons, microbiologists, and pharmacists has to be assembled at various points in time during the diagnostic process, the treatment planning, and execution of said treatment including its surveillance and follow-up care. To efficiently execute such a team effort with minimal loss of information and limit the amount of time spent on the organization and logistics of the team meetings and documentation, an IT platform was established enabling the metachronous and collaborative work on each case. While the design and implementation processes were time consuming, and the input of relevant information by each specialist, even though based on the division of labor, is elaborate, the use of the platform has increased team meeting efficiency and availability of relevant information.

\section{Authors' Contributions}

Conception and design of the study: CS, JT, RER, DB, RB, HM; Generation and collection of the data: CS, SF, PR, CQ, JT; Assembly and/or analysis of the data: CS, SF, PR, CQ, JT, HM; Drafting and revising the manuscript: CS, HM, FP; Approval of the final version of the manuscript: All authors read and approved the final manuscript.

\section{Ethics Approval and Consent to Participate}

Ethics approval was given by our institutional ethics review board under no. 195/18 S.

\section{Conflicts of Interest}

None of the authors have any competing interests.

\section{Funding}

This work was not funded externally.

\section{Acknowledgements}

The Authors would like to thank Silke Melzner for her invaluable support in the organization of their infections board meetings.

\section{References}

1 Kurtz S, Ong K, Lau E, Mowat F and Halpern M: Projections of primary and revision hip and knee arthroplasty in the united states from 2005 to 2030. J Bone Joint Surg Am 89(4): 780-785, 2007. PMID: 17403800. DOI: 10.2106/JBJS.F.00222

2 Kurtz SM, Lau E, Schmier J, Ong KL, Zhao K and Parvizi J: Infection burden for hip and knee arthroplasty in the united states. J Arthroplasty 23(7): 984-991, 2008. PMID: 18534466. DOI: $10.1016 /$ j.arth.2007.10.017

3 Parvizi J, Zmistowski B, Berbari EF, Bauer TW, Springer BD, Della Valle CJ, Garvin KL, Mont MA, Wongworawat MD and Zalavras CG: New definition for periprosthetic joint infection: From the workgroup of the musculoskeletal infection society. Clin Orthop Relat Res 469(11): 2992-2994, 2011. PMID: 21938532. DOI: $10.1007 / \mathrm{s} 11999-011-2102-9$

4 Zmistowski B, Della Valle C, Bauer TW, Malizos KN, Alavi A, Bedair H, Booth RE, Choong P, Deirmengian C, Ehrlich GD, Gambir A, Huang R, Kissin Y, Kobayashi H, Kobayashi N, Krenn V, Lorenzo D, Marston SB, Meermans G, Perez J, Ploegmakers JJ, Rosenberg A, Simpfendorfer C, Thomas P, Tohtz S, Villafuerte JA, Wahl P, Wagenaar FC and Witzo E: Diagnosis of periprosthetic joint infection. J Orthop Res 32 Suppl 1: S98-107, 2014. PMID: 24464903. DOI: $10.1002 /$ jor.22553

5 Springer BD: The diagnosis of periprosthetic joint infection. J Arthroplasty 30(6): 908-911, 2015. PMID: 25913561. DOI: 10.1016/j.arth.2015.03.042

6 Parvizi J, Adeli B, Zmistowski B, Restrepo C and Greenwald AS: Management of periprosthetic joint infection: The current knowledge: Aaos exhibit selection. J Bone Joint Surg Am 94(14): e104, 2012. PMID: 22810411. DOI: 10.2106/JBJS. K.01417

7 Horan TC, Gaynes RP, Martone WJ, Jarvis WR and Emori TG: Cdc definitions of nosocomial surgical site infections, 1992: A modification of cdc definitions of surgical wound infections. Infect Control Hosp Epidemiol 13(10): 606-608, 1992. PMID: 1334988.

8 Parvizi J and Della Valle CJ: AAOS clinical practice guideline: Diagnosis and treatment of periprosthetic joint infections of the hip and knee. J Am Acad Orthop Surg 18(12): 771-772, 2010. PMID: 21119143.

9 Uckay I, Lubbeke A, Emonet S, Tovmirzaeva L, Stern R, Ferry T, Assal M, Bernard L, Lew D and Hoffmeyer P: Low incidence of haematogenous seeding to total hip and knee prostheses in patients with remote infections. J Infect 59(5): 337-345, 2009. PMID: 19732794. DOI: 10.1016/j.jinf.2009.08.015

10 Portillo ME, Salvado M, Alier A, Sorli L, Martinez S, Horcajada JP and Puig L: Prosthesis failure within 2 years of implantation is highly predictive of infection. Clin Orthop Relat Res 471(11): 36723678, 2013. PMID: 23904245. DOI: 10.1007/s11999-013-3200-7

11 Schinsky MF, Della Valle CJ, Sporer SM and Paprosky WG: Perioperative testing for joint infection in patients undergoing revision total hip arthroplasty. J Bone Joint Surg Am 90(9): 18691875, 2008. PMID: 18762646. DOI: 10.2106/JBJS.G. 01255

12 Zmistowski B, Restrepo C, Huang R, Hozack WJ and Parvizi J: Periprosthetic joint infection diagnosis: a complete understanding of 
white blood cell count and differential. J Arthroplasty 27(9): 15891593, 2012. PMID: 22543180. DOI: 10.1016/j.arth.2012.03.059

13 Fink B, Gebhard A, Fuerst M, Berger I and Schafer P: High diagnostic value of synovial biopsy in periprosthetic joint infection of the hip. Clin Orthop Relat Res 471(3): 956-964, 2013. PMID: 22806261. DOI: 10.1007/s11999-012-2474-5

14 Collins SA, Bavuso K, Zuccotti G and Rocha RA: Lessons learned for collaborative clinical content development. Appl Clin Inform 4(2): 304-316, 2013. PMID: 23874366. DOI: 10.4338/ ACI-2013-02-CR-0014

15 Marks M, Pollara G, Miller D, Bhatt S, Reynolds C, Gant V and Noursadeghi M: elCID: An electronic clinical infection database to support integrated clinical services and research in infectious diseases. J Infect 71(3): 402-405, 2015. PMID: 25892135. DOI: 10.1016/j.jinf.2015.04.007

16 Vehreschild JJ, Morgen G, Cornely OA, Hartmann P, Koch S, Kalka-Moll W, Wyen C, Vehreschild MJ, Lehmann C, Gillor D, Seifert H, Kremer G, Fatkenheuer G and Jung N: Evaluation of an infectious disease consultation programme in a german tertiary care hospital. Infection 41(6): 1121-1128, 2013. PMID: 23925637. DOI: $10.1007 / \mathrm{s} 15010-013-0512-1$

17 Evans RS, Pestotnik SL, Classen DC, Clemmer TP, Weaver LK, Orme JF Jr., Lloyd JF and Burke JP: A computer-assisted management program for antibiotics and other antiinfective agents. N Engl J Med 338(4): 232-238, 1998. PMID: 9435330. DOI: 10.1056/NEJM199801223380406

18 Rocchio BJ: Achieving cost reduction through data analytics. AORN J 104(4): 320-325, 2016. PMID: 27692078. DOI: 10.1016/ j.aorn.2016.07.010.

19 Hartzband D and Jacobs F: Deployment of analytics into the healthcare safety net: Lessons learned. Online J Public Health Inform 8(3): e203, 2016. PMID: 28210424. DOI: 10.5210/ ojphi.v8i3.7000

20 Ruchholtz S, Lewan U, Debus F, Mand C, Siebert H and Kuhne CA: Traumanetzwerk $\operatorname{dgu}((\mathrm{r}))$ : optimizing patient flow and management. Injury 45(Suppl 3): S89-92, 2014. PMID: 25284242. DOI: $10.1016 /$ j.injury.2014.08.024
21 Frink M, Kuhne C, Debus F, Pries A and Ruchholtz S: [the traumanetzwerk dgu project. Goals, conception, and successes achieved]. Unfallchirurg 116(1): 61-71, 2013. PMID: 23307433. DOI: $10.1007 / \mathrm{s} 00113-012-2326-5$

22 Pan E, Cusack C, Hook J, Vincent A, Kaelber DC, Bates DW and Middleton B: The value of provider-to-provider telehealth. Telemed J E Health 14(5): 446-453, 2008. PMID: 18578679. DOI: $10.1089 / \mathrm{tmj} .2008 .0017$

23 Akiyama $\mathrm{M}$ and Yoo BK: A systematic review of the economic evaluation of telemedicine in Japan. J Prev Med Public Health 49(4): 183-196, 2016. PMID: 27499161. DOI: 10.3961/jpmph. 16.043

24 Huber-Wagner S, Lefering R, Qvick LM, Korner M, Kay MV, Pfeifer KJ, Reiser M, Mutschler W and Kanz KG: Effect of whole-body ct during trauma resuscitation on survival: A retrospective, multicentre study. Lancet 373(9673): 1455-1461, 2009. PMID: 19321199. DOI: 10.1016/S0140-6736(09)60232-4

25 Muhlhofer HM, Kanz KG, Pohlig F, Lenze U, Lenze F, Toepfer A, von Eisenhart-Rothe R and Schauwecker J: Implementation of an algorithm for prosthetic joint infection: Deviations and problems. Surg Infect (Larchmt) 18(2): 164-169, 2017. PMID: 27925502. DOI: 10.1089/sur.2015.208

26 Armstrong MD, Carli AV, Abdelbary H, Poitras S, Lapner P and Beaule PE: Tertiary care centre adherence to unified guidelines for management of periprosthetic joint infections: a gap analysis. Can J Surg 60(6): 8617, 2017. PMID: 29171826. DOI: 10.1503/ cjs.008617
Received May 1, 2019

Revised June 14, 2019

Accepted June 28, 2019 